\title{
New potential condition on homoclinic orbits for a class of discrete Hamiltonian systems
}

\section{Xiaoping Wang*}

\section{*Correspondence:}

wxp31415@sina.com

Department of Mathematics,

Xiangnan College, Chenzhou,

Hunan 423000, P.R. China

\begin{abstract}
In the present paper, we establish an existence criterion to guarantee that the second-order self-adjoint discrete Hamiltonian system

$\Delta[p(n) \Delta u(n-1)]-L(n) u(n)+\nabla W(n, u(n))=0$ has a nontrivial homoclinic solution, which does not need periodicity and coercivity conditions on $L(n)$.
\end{abstract}

MSC: 39A11; 58E05; 70H05

Keywords: homoclinic solution; discrete Hamiltonian system; critical point

\section{Introduction}

Consider the second-order self-adjoint discrete Hamiltonian system

$$
\triangle[p(n) \triangle u(n-1)]-L(n) u(n)+\nabla W(n, u(n))=0,
$$

where $n \in \mathbb{Z}, u \in \mathbb{R}^{\mathcal{N}}, \Delta u(n)=u(n+1)-u(n)$ is the forward difference, $p, L: \mathbb{Z} \rightarrow \mathbb{R}^{\mathcal{N} \times \mathcal{N}}$ and $W: \mathbb{Z} \times \mathbb{R}^{\mathcal{N}} \rightarrow \mathbb{R}$.

Discrete Hamiltonian systems can be applied in many areas, such as physics, chemistry, and so on. For more discussions on discrete Hamiltonian systems, we refer the reader to $[1,2]$.

As usual, we say that a solution $u(n)$ of system (1.1) is homoclinic (to 0$)$ if $u(n) \rightarrow 0$ as $n \rightarrow \pm \infty$. In addition, if $u(n) \not \equiv 0$ then $u(n)$ is called a nontrivial homoclinic solution.

The existence and multiplicity of homoclinic solutions of system (1.1) or its special forms have been investigated by many authors. Papers [3-8] deal with the periodic case where $p, L$ and $W$ are periodic in $n$ or independent of $n$. In contrast, if the periodicity is lost, because of lack of compactness of the Sobolev embedding, up to our knowledge, all existence results require a coercivity condition on $L$ :

$$
\lim _{|n| \rightarrow \infty}\left[\inf _{x \in \mathbb{R}^{\mathcal{N}},|x|=1}(L(n) x, x)\right]=\infty
$$

For example, see [9-14]. In the above mentioned papers, except [14], $L$ was always required to be positive definite.

In this paper, we derive an existence result which does not need periodicity and coercivity conditions on $L(n)$. To state our results precisely, we make the following assumptions.

(P) $p(n)$ is $\mathcal{N} \times \mathcal{N}$ real symmetric positive definite matrix for all $n \in \mathbb{Z}$. 
(L) $L(n)$ is $\mathcal{N} \times \mathcal{N}$ real symmetric nonnegative definite matrix for all $n \in \mathbb{Z}$, and there exist a positive integer $N_{0} \in \mathbb{Z}$ and $\beta>0$ such that

$$
\min _{x \in \mathbb{R}^{\mathcal{N}},|x|=1}(L(n) x, x) \geq \beta, \quad|n| \geq N_{0}
$$

where here and in the sequel, $(\cdot, \cdot)$ denotes the standard inner product in $\mathbb{R}^{\mathcal{N}}$ and $|\cdot|$ is the induced norm.

(W1) $W(n, x)$ is continuously differentiable in $x$ for every $n \in \mathbb{Z}, W(n, 0)=0$,

$$
W(n, x) \geq 0 \text { for all }(n, x) \in \mathbb{Z} \times \mathbb{R}^{\mathcal{N}} .
$$

(W2) $\lim _{|x| \rightarrow 0} \frac{\nabla W(n, x)}{|x|}=0$ uniformly for all $n \in \mathbb{Z}$.

(W3) $\lim _{|x| \rightarrow \infty} \frac{|W(n, x)|}{|x|^{2}}=\infty$ uniformly for all $n \in \mathbb{Z}$.

(W4) $\widetilde{W}(n, x):=\frac{1}{2}(\nabla W(n, x), x)-W(n, x) \geq 0, \forall(n, x) \in \mathbb{Z} \times \mathbb{R}^{\mathcal{N}}$, and there exist $\varepsilon \in(0,1), c_{0}>0$, and $R_{0}>0$ such that

$$
(\nabla W(n, x), x) \leq \frac{\beta(1-\varepsilon)}{2}|x|^{2}, \quad \forall(n, x) \in \mathbb{Z} \times \mathbb{R}^{\mathcal{N}},|x| \leq R_{0}
$$

and

$$
(\nabla W(n, x), x) \leq c_{0}|x|^{2} \widetilde{W}(n, x), \quad \forall(n, x) \in \mathbb{Z} \times \mathbb{R}^{\mathcal{N}},|x| \geq R_{0}
$$

Now, we are ready to state the main result of this paper.

Theorem 1.1 Assume that $p, L$ and W satisfy (P), (L), (W1), (W2), (W3), and (W4). If there exist $n_{0} \in \mathbb{Z}$ and $x_{0} \in \mathbb{R}^{\mathcal{N}}$ such that

$$
\beta \geq 2 c_{0} \sup _{s \geq 0}\left[\frac{s^{2}}{2}\left(\left(p\left(n_{0}\right)+p\left(n_{0}+1\right)+L\left(n_{0}\right)\right) x_{0}, x_{0}\right)-W\left(n_{0}, s x_{0}\right)\right],
$$

then system (1.1) possesses a nontrivial homoclinic solution.

In Theorem 1.1, we replace (L) and (W4) by the following assumptions:

$\left(\mathrm{L}^{\prime}\right) \quad L(n)$ is $\mathcal{N} \times \mathcal{N}$ real symmetric nonnegative definite matrix for all $n \in \mathbb{Z}$, and it satisfies (1.2)

$\left(\mathrm{W} 4^{\prime}\right) \widetilde{W}(n, x):=\frac{1}{2}(\nabla W(n, x), x)-W(n, x) \geq 0, \forall(n, x) \in \mathbb{Z} \times \mathbb{R}^{\mathcal{N}}$, and there exist $c_{0}>0$ and $R_{0}>0$ such that

$$
(\nabla W(n, x), x) \leq c_{0}|x|^{2} \widetilde{W}(n, x), \quad \forall(n, x) \in \mathbb{Z} \times \mathbb{R}^{\mathcal{N}},|x| \geq R_{0} .
$$

Then we have the following corollary immediately.

Corollary 1.2 Assume that $p, L$ and W satisfy (P), (L'), (W1), (W2), (W3) and (W4'). Then system (1.1) possesses a nontrivial homoclinic solution.

Remark 1.3 If $W(n, x)$ satisfies the well-known global Ambrosetti-Rabinowitz superquadratic condition: 
(AR) there exists $\mu>2$ such that

$$
0<\mu W(n, x) \leq(\nabla W(n, x), x), \quad \forall(n, x) \in \mathbb{Z} \times \mathbb{R}^{\mathcal{N}} \backslash\{0\},
$$

then there exists a constant $C_{0}>0$ such that

$$
W(n, x) \geq C_{0}|x|^{\mu}, \quad \forall(n, x) \in \mathbb{Z} \times \mathbb{R}^{\mathcal{N}},|x| \geq 1
$$

moreover $\widetilde{W}(n, x)>0$ for all $(n, x) \in \mathbb{Z} \times\left(\mathbb{R}^{\mathcal{N}} \backslash\{0\}\right)$, and

$$
(\nabla W(n, x), x) \leq \frac{2 \mu}{\mu-2}|x|^{2} \widetilde{W}(n, x), \quad \forall(n, x) \in \mathbb{Z} \times \mathbb{R}^{\mathcal{N}},|x| \geq 1 .
$$

In addition, by virtue of (W2), there exists $\beta_{1}>0$ such that

$$
(\nabla W(n, x), x) \leq \frac{\beta_{1}}{2}|x|^{2}, \quad \forall(n, x) \in \mathbb{Z} \times \mathbb{R}^{\mathcal{N}},|x| \leq 1 .
$$

These show that (W3) and (W4) hold with $R_{0}=1, c_{0}=2 \mu /(\mu-2)$ and $\beta>\beta_{1}$. Let $p(n)=$ $I_{\mathcal{N}}$ and $L(n)=\lambda n^{2} /\left(1+n^{2}\right) I_{\mathcal{N}}$ and choose $n_{0}=0$ and $x_{0}=(1,0, \ldots, 0) \in \mathbb{R}^{\mathcal{N}}$. In view of Theorem 1.1, if

$$
\lambda>\max \left\{\frac{4 \mu}{\mu-2} \sup _{s \geq 0}\left[s^{2}-W\left(0, s x_{0}\right)\right], \beta_{1}\right\},
$$

then system (1.1) possesses a nontrivial homoclinic solution.

Example 1.4 Let $p(n)=I_{\mathcal{N}}, L(n)=\left[1+\lambda n^{2} /\left(1+n^{2}\right)\right] I_{\mathcal{N}}$ and

$$
W(n, x)=|x|^{2} \ln \left(1+|x|^{2}\right) .
$$

Then

$$
(\nabla W(n, x), x)=2|x|^{2} \ln \left(1+|x|^{2}\right)+\frac{2|x|^{4}}{1+|x|^{2}} .
$$

It is easy to see that $\widetilde{W}(n, x) \geq 0$ for all $(n, x) \in \mathbb{Z} \times \mathbb{R}^{\mathcal{N}}$, and

$$
\begin{aligned}
& (\nabla W(n, x), x) \leq(2 \ln 2+1)|x|^{2}, \quad \forall(n, x) \in \mathbb{Z} \times \mathbb{R}^{\mathcal{N}},|x| \leq 1, \\
& (\nabla W(n, x), x) \leq 6|x|^{2} \widetilde{W}(n, x), \quad \forall(n, x) \in \mathbb{Z} \times \mathbb{R}^{\mathcal{N}},|x| \geq 1 .
\end{aligned}
$$

These show that (W3) and (W4) hold with $R_{0}=1, c_{0}=6$ and $\beta>2(2 \ln 2+1)$. We choose $n_{0}=0$ and $x_{0}=(1,0, \ldots, 0) \in \mathbb{R}^{\mathcal{N}}$. Then

$$
\begin{aligned}
\sup _{s \geq 0} & {\left[\frac{s^{2}}{2}\left(\left(p\left(n_{0}\right)+p\left(n_{0}+1\right)+L\left(n_{0}\right)\right) x_{0}, x_{0}\right)-W\left(n_{0}, s x_{0}\right)\right] } \\
\quad= & \sup _{s \geq 0}\left[\frac{3 s^{2}}{2}-s^{2} \ln \left(1+s^{2}\right)\right]<6-\ln 2 .
\end{aligned}
$$

In view of Theorem 1.1, if $\lambda \geq 12(6-\ln 2)$, then system (1.1) possesses a nontrivial homoclinic solution. 


\section{Preliminaries}

Throughout this section, we always assume that $p$ and $L$ satisfy (P) and (L). Let

$$
\begin{aligned}
& S=\left\{\{u(n)\}_{n \in \mathbb{Z}}: u(n) \in \mathbb{R}^{\mathcal{N}}, n \in \mathbb{Z}\right\}, \\
& E=\left\{u \in S: \sum_{n \in \mathbb{Z}}[(p(n+1) \triangle u(n), \Delta u(n))+(L(n) u(n), u(n))]<+\infty\right\},
\end{aligned}
$$

and for $u, v \in E$, let

$$
\langle u, v\rangle=\sum_{n \in \mathbb{Z}}[(p(n+1) \Delta u(n), \Delta v(n))+(L(n) u(n), v(n))] .
$$

Then $E$ is a Hilbert space with the above inner product, and the corresponding norm is

$$
\|u\|=\left\{\sum_{n \in \mathbb{Z}}[(p(n+1) \Delta u(n), \Delta u(n))+(L(n) u(n), u(n))]\right\}^{1 / 2}, \quad u \in E .
$$

As usual, for $1 \leq s<+\infty$, set

$$
l^{s}\left(\mathbb{Z}, \mathbb{R}^{\mathcal{N}}\right)=\left\{u \in S: \sum_{n \in \mathbb{Z}}|u(n)|^{s}<+\infty\right\}
$$

and

$$
l^{\infty}\left(\mathbb{Z}, \mathbb{R}^{\mathcal{N}}\right)=\left\{u \in S: \sup _{n \in \mathbb{Z}}|u(n)|<+\infty\right\},
$$

and their norms are defined by

$$
\begin{aligned}
& \|u\|_{s}=\left(\sum_{n \in \mathbb{Z}}|u(n)|^{s}\right)^{1 / s}, \quad \forall u \in l^{s}\left(\mathbb{Z}, \mathbb{R}^{\mathcal{N}}\right) ; \\
& \|u\|_{\infty}=\sup _{n \in \mathbb{Z}}|u(n)|, \quad \forall u \in l^{\infty}\left(\mathbb{Z}, \mathbb{R}^{\mathcal{N}}\right),
\end{aligned}
$$

respectively.

Lemma 2.1 Suppose that (L) is satisfied. Then

$$
\|u\|_{\infty} \leq \frac{1}{\sqrt{\beta}}\|u\|+\sum_{|s| \leq N_{0}-1}|\Delta u(s)|, \quad u \in E
$$

and

$$
\|u\|_{\infty} \leq \max \left\{\sqrt{\frac{2}{\beta}}, \sqrt{\frac{2 N_{0}}{\alpha}}\right\}\|u\|, \quad u \in E
$$

where $\alpha=\min _{|n| \leq N_{0},|x|=1}(p(n) x, x)$. 
Proof Since $u \in E$, it follows that $\lim _{|n| \rightarrow \infty}|u(n)|=0$. Hence, there exists $n_{*} \in \mathbb{Z}$ such that $\|u\|_{\infty}=\left|u\left(n_{*}\right)\right|$. There are two possible cases.

Case (i). $\left|n_{*}\right| \geq N_{0}$. According to (L), one has

$$
\|u\|_{\infty}^{2}=\left|u\left(n_{*}\right)\right|^{2} \leq \frac{1}{\beta} \sum_{|s| \geq N_{0}}(L(s) u(s), u(s)) \leq \frac{1}{\beta}\|u\|
$$

Case (ii). $\left|n_{*}\right|<N_{0}$. Without loss of generality, we can assume that $n_{*} \geq 0$, then

$$
\begin{aligned}
\|u\|_{\infty} & \leq\left|u\left(N_{0}\right)\right|+\sum_{s=n_{*}}^{N_{0}-1}|\Delta u(s)| \\
& \leq\left[\frac{1}{\beta} \sum_{|s| \geq N_{0}}(L(s) u(s), u(s))\right]^{1 / 2}+\sqrt{\frac{N_{0}}{\alpha}}\left(\sum_{s=n_{*}}^{N_{0}-1} \alpha|\Delta u(s)|^{2}\right)^{1 / 2} \\
& \leq \sqrt{2}\left[\frac{1}{\beta} \sum_{|s| \geq N_{0}}(L(s) u(s), u(s))+\frac{N_{0}}{\alpha} \sum_{s=n_{*}}^{N_{0}-1}(p(s+1) \Delta u(s), \Delta u(s))\right]^{1 / 2} \\
& \leq \max \left\{\sqrt{\frac{2}{\beta}}, \sqrt{\frac{2 N_{0}}{\alpha}}\right\}\|u\| .
\end{aligned}
$$

Cases (i) and (ii) imply that (2.1) and (2.2) hold.

Now we define a functional $\Phi$ on $E$ by

$$
\Phi(u)=\frac{1}{2} \sum_{n \in \mathbb{Z}}[(p(n+1) \Delta u(n), \Delta u(n))+(L(n) u(n), u(n))]-\sum_{n \in \mathbb{Z}} W(n, u(n)) .
$$

For any $u \in E$, there exists an $n_{1} \in \mathbb{N}$ such that $|u(n)| \leq 1$ for $|n| \geq n_{1}$. Hence, under assumptions (P), (L), (W1), and (W2), the functional $\Phi$ is of class $C^{1}(E, \mathbb{R})$. Moreover,

$$
\Phi(u)=\frac{1}{2}\|u\|^{2}-\sum_{n \in \mathbb{Z}} W(n, u), \quad \forall u \in E
$$

and

$$
\left\langle\Phi^{\prime}(u), v\right\rangle=\langle u, v\rangle-\sum_{n \in \mathbb{Z}}(\nabla W(n, u), v), \quad \forall u, v \in E
$$

Furthermore, the critical points of $\Phi$ in $E$ are solutions of system (1.1) with $u( \pm \infty)=0$, see $[5,6]$.

Let $e=\{e(n)\}_{n \in \mathbb{Z}} \in E$ with $e\left(n_{0}\right)=x_{0}$ and $e(n)=0 \in \mathbb{R}^{\mathcal{N}}$ for $n \neq n_{0}$.

Lemma 2.2 Suppose that (L), (W1) and (W2) are satisfied. Then

$$
\sup \{\Phi(s e): s \geq 0\} \leq \sup _{s \geq 0}\left[\frac{s^{2}}{2}\left(\left(p\left(n_{0}\right)+p\left(n_{0}+1\right)+L\left(n_{0}\right)\right) x_{0}, x_{0}\right)-W\left(n_{0}, s x_{0}\right)\right] .
$$


Proof From (2.4) and the definition of $e$, we get

$$
\begin{aligned}
\Phi(s e)= & \frac{s^{2}}{2} \sum_{n \in \mathbb{Z}}[(p(n+1) \triangle e(n), \triangle e(n))+(L(n) e(n), e(n))]-\sum_{n \in \mathbb{Z}} W(n, s e(n)) \\
= & \frac{s^{2}}{2}\left[\left(p\left(n_{0}+1\right) \triangle e\left(n_{0}\right), \triangle e\left(n_{0}\right)\right)+\left(p\left(n_{0}\right) \triangle e\left(n_{0}-1\right), \triangle e\left(n_{0}-1\right)\right)\right. \\
& \left.+\left(L\left(n_{0}\right) e\left(n_{0}\right), e\left(n_{0}\right)\right)\right]-W\left(n_{0}, s e\left(n_{0}\right)\right) \\
= & \frac{s^{2}}{2}\left(\left(p\left(n_{0}\right)+p\left(n_{0}+1\right)+L\left(n_{0}\right)\right) x_{0}, x_{0}\right)-W\left(n_{0}, s x_{0}\right) .
\end{aligned}
$$

Now the conclusion of Lemma 2.1 follows by (2.8).

Applying the mountain-pass lemma without the (PS) condition, by standard arguments, we can prove the following lemma.

Lemma 2.3 Let $W(n, x) \geq 0, \forall(n, x) \in \mathbb{Z} \times \mathbb{R}^{\mathcal{N}}$. Suppose that (P), (L), (W1), (W2) and (W3) are satisfied. Then there exist a constant $c \in\left(0, \sup _{s \geq 0} \Phi(s e)\right]$ and a sequence $\left\{u_{k}\right\} \subset E$ satisfying

$$
\Phi\left(u_{k}\right) \rightarrow c, \quad\left\|\Phi^{\prime}\left(u_{k}\right)\right\|\left(1+\left\|u_{k}\right\|\right) \rightarrow 0
$$

Lemma 2.4 Suppose that (P), (L), (W1), (W2), (W3), and (W4) are satisfied. Then any sequence $\left\{u_{k}\right\} \subset$ E satisfying

$$
\Phi\left(u_{k}\right) \rightarrow c>0, \quad\left\langle\Phi^{\prime}\left(u_{k}\right), u_{k}\right\rangle \rightarrow 0
$$

is bounded in $E$.

Proof To prove the boundedness of $\left\{u_{k}\right\}$, arguing by contradiction, suppose that $\left\|u_{k}\right\| \rightarrow$ $\infty$. Let $v_{k}=u_{k} /\left\|u_{k}\right\|$. Then $\left\|v_{k}\right\|=1$. By virtue of (2.5), (2.6), and (2.10), we have

$$
\Phi\left(u_{k}\right)-\frac{1}{2}\left\langle\Phi^{\prime}\left(u_{k}\right), u_{k}\right\rangle=\sum_{n \in \mathbb{Z}} \widetilde{W}\left(n, u_{k}\right)=c+o(1) .
$$

If $\delta:=\lim \sup _{k \rightarrow \infty}\left\|v_{k}\right\|_{\infty}=0$, then it follows from (L), (W4) and (2.11) that

$$
\begin{aligned}
\sum_{\left|u_{k}\right|<R_{0}}\left|\left(\nabla W\left(n, u_{k}\right), u_{k}\right)\right| & \leq \frac{\beta}{2} \sum_{\left|u_{k}\right|<R_{0}}\left|u_{k}\right|^{2} \leq \frac{\beta}{2} \sum_{|s| \geq N_{0}}\left|u_{k}(s)\right|^{2}+\frac{\beta}{2} \sum_{|s|<N_{0}}\left|u_{k}(s)\right|^{2} \\
& \leq \frac{1}{2}\left\|u_{k}\right\|^{2}+N_{0} \beta\left\|u_{k}\right\|^{2}\left\|v_{k}\right\|_{\infty}^{2} \leq\left[\frac{1}{2}+o(1)\right]\left\|u_{k}\right\|^{2}
\end{aligned}
$$

and

$$
\begin{aligned}
\sum_{\left|u_{k}\right| \geq R_{0}} \frac{\left|\left(\nabla W\left(n, u_{k}\right), u_{k}\right)\right|}{\left\|u_{k}\right\|^{2}} & \leq c_{0} \sum_{\left|u_{k}\right| \geq R_{0}}\left|v_{k}\right|^{2} \widetilde{W}\left(n, u_{k}\right) \leq c_{0}\left\|v_{k}\right\|_{\infty}^{2} \sum_{\left|u_{k}\right| \geq R_{0}} \widetilde{W}\left(n, u_{k}\right) \\
& \leq c_{0}(c+1)\left\|v_{k}\right\|_{\infty}^{2} \rightarrow 0, \quad k \rightarrow \infty .
\end{aligned}
$$


Combining (2.12) with (2.13) and using (2.5) and (2.10), we have

$$
\begin{aligned}
1+o(1) & \leq \frac{\left\|u_{k}\right\|^{2}-\left\langle\Phi^{\prime}\left(u_{k}\right), u_{k}\right\rangle}{\left\|u_{k}\right\|^{2}} \leq \sum_{n \in \mathbb{Z}} \frac{\left|\left(\nabla W\left(n, u_{k}\right), u_{k}\right)\right|}{\left\|u_{k}\right\|^{2}} \\
& =\sum_{\left|u_{k}\right|<R_{0}} \frac{\left|\left(\nabla W\left(n, u_{k}\right), u_{k}\right)\right|}{\left\|u_{k}\right\|^{2}}+\sum_{\left|u_{k}\right| \geq R_{0}} \frac{\left|\left(\nabla W\left(n, u_{k}\right), u_{k}\right)\right|}{\left\|u_{k}\right\|^{2}} \leq \frac{1}{2}+o(1) .
\end{aligned}
$$

This contradiction shows that $\delta>0$.

Going if necessary to a subsequence, we may assume the existence of $n_{k} \in \mathbb{Z}$ such that

$$
\left|v_{k}\left(n_{k}\right)\right|=\left\|v_{k}\right\|_{\infty}>\frac{\delta}{2}
$$

Let $w_{k}(n)=v_{k}\left(n+n_{k}\right)$, then

$$
\left|w_{k}(0)\right|>\frac{\delta}{2}, \quad \forall k \in \mathbb{N}
$$

Now we define $\tilde{u}_{k}(n)=u_{k}\left(n+n_{k}\right)$. Then $\tilde{u}_{k}(n) /\left\|u_{k}\right\|=w_{k}(n)$ and $\left\|w_{k}\right\|_{2}=\left\|v_{k}\right\|_{2}$. Passing to a subsequence, we have $w_{k} \rightarrow w$ in $l^{2}\left(\mathbb{Z}, \mathbb{R}^{\mathcal{N}}\right)$, then $w_{k}(n) \rightarrow w(n)$ for all $n \in \mathbb{Z}$. Clearly, (2.15) implies that $w(0) \neq 0$.

It is obvious that $w(n) \neq 0$ implies $\lim _{k \rightarrow \infty}\left|\tilde{u}_{k}(n)\right|=\infty$. Hence, it follows from (2.5), (2.10), and (W3) that

$$
\begin{aligned}
0 & =\lim _{k \rightarrow \infty} \frac{c+o(1)}{\left\|u_{k}\right\|^{2}}=\lim _{k \rightarrow \infty} \frac{\Phi\left(u_{k}\right)}{\left\|u_{k}\right\|^{2}} \\
& =\lim _{k \rightarrow \infty}\left[\frac{1}{2}-\sum_{n \in \mathbb{Z}} \frac{W\left(n, u_{k}\right)}{\left|u_{k}\right|^{2}}\left|v_{k}\right|^{2}\right] \\
& =\lim _{k \rightarrow \infty}\left[\frac{1}{2}-\sum_{n \in \mathbb{Z}} \frac{W\left(n+k_{n}, \tilde{u}_{k}\right)}{\left|\tilde{u}_{k}\right|^{2}}\left|w_{k}\right|^{2}\right] \\
& \leq \frac{1}{2}-\liminf _{k \rightarrow \infty} \sum_{n \in \mathbb{Z}} \frac{W\left(n+k_{n}, \tilde{u}_{k}\right)}{\left|\tilde{u}_{k}\right|^{2}}\left|w_{k}\right|^{2} \\
& =-\infty,
\end{aligned}
$$

which is a contradiction. Thus $\left\{u_{k}\right\}$ is bounded in $E$.

\section{Proof of theorem}

Proof of Theorem 1.1 Applying Lemmas 2.3 and 2.4, we deduce that there exists a bounded sequence $\left\{u_{k}\right\} \subset E$ satisfying (2.9). By Lemma 2.2 and (1.3), one has

$$
c \leq \frac{\beta}{2 c_{0}}
$$

Going if necessary to a subsequence, we can assume that $u_{k} \rightarrow \bar{u}$ in $E$ and $\Phi^{\prime}\left(u_{k}\right) \rightarrow 0$. Next, we prove that $\bar{u} \neq 0$. 
Arguing by contradiction, suppose that $\bar{u}=0$, i.e. $u_{k} \rightarrow 0$ in $E$, and so $u_{k}(n) \rightarrow 0$ for every $n \in \mathbb{Z}$. Hence,

$$
\left\|u_{k}\right\|_{2}^{2}=\sum_{|n| \geq N_{0}}\left|u_{k}(n)\right|^{2}+\sum_{|n|<N_{0}}\left|u_{k}(n)\right|^{2} \leq \frac{1}{\beta}\left\|u_{k}\right\|^{2}+o(1) .
$$

According to (W4) and (3.2), one gets

$$
\sum_{\left|u_{k}\right|<R_{0}}\left(\nabla W\left(n, u_{k}\right), u_{k}\right) \leq \frac{\beta(1-\varepsilon)}{2} \sum_{\left|u_{k}\right|<R_{0}}\left|u_{k}\right|^{2} \leq \frac{1-\varepsilon}{2}\left\|u_{k}\right\|^{2}+o(1) .
$$

By virtue of (2.5), (2.6), and (2.9), we have

$$
\Phi\left(u_{k}\right)-\frac{1}{2}\left\langle\Phi^{\prime}\left(u_{k}\right), u_{k}\right\rangle=\sum_{n \in \mathbb{Z}} \widetilde{W}\left(n, u_{k}\right)=c+o(1) .
$$

Using (W4), (2.1), (3.1), (3.2), and (3.4), we obtain

$$
\begin{aligned}
\sum_{\left|u_{k}\right| \geq R_{0}}\left(\nabla W\left(n, u_{k}\right), u_{k}\right) & \leq c_{0} \sum_{\left|u_{k}\right| \geq R_{0}}\left|u_{k}\right|^{2} \widetilde{W}\left(n, u_{k}\right) \\
& \leq c_{0}\left\|u_{k}\right\|_{\infty}^{2} \sum_{\left|u_{k}\right| \geq R_{0}} \widetilde{W}\left(n, u_{k}\right) \\
& \leq c_{0} c\left\|u_{k}\right\|_{\infty}^{2}+o(1) \\
& \leq c_{0} c\left(\frac{1}{\sqrt{\beta}}\left\|u_{k}\right\|+\sum_{|s| \leq N_{0}-1}\left|\Delta u_{k}(s)\right|\right)^{2}+o(1) \\
& =\frac{c_{0} c}{\beta}\left\|u_{k}\right\|^{2}+o(1) \\
& \leq \frac{1}{2}\left\|u_{k}\right\|^{2}+o(1),
\end{aligned}
$$

which, together with (2.6), (2.9), and (3.3), yields

$$
\begin{aligned}
o(1) & =\left\langle\Phi^{\prime}\left(u_{k}\right), u_{k}\right\rangle=\left\|u_{k}\right\|^{2}-\sum_{n \in \mathbb{Z}}\left(\nabla W\left(n, u_{k}\right), u_{k}\right) \\
& \geq \frac{\varepsilon}{2}\left\|u_{k}\right\|^{2}+o(1),
\end{aligned}
$$

resulting in the fact that $\left\|u_{k}\right\| \rightarrow 0$. Consequently, it follows from (W1), (2.5), and (2.9) that

$$
0<c=\lim _{k \rightarrow \infty} \Phi\left(u_{k}\right)=\Phi(0)=0 .
$$

This contradiction shows $\bar{u} \neq 0$. By standard arguments, we easily prove that $\bar{u}$ is a nontrivial solution of (1.1). 


\section{Acknowledgements}

This work is partially supported by Scientific Research Fund of Hunan Provincial Education Department $(13 \mathrm{A093}, 07 \mathrm{A066})$ and supported by Hunan Provincial Natural Science Foundation of China (No. 14JJ2133).

Received: 15 December 2013 Accepted: 10 February 2014 Published: 21 Feb 2014

\section{References}

1. Agarwal, RP: Difference Equations and Inequalities: Theory, Methods, and Applications, 2nd edn. Dekker, New York (2000)

2. Ahlbran, CD, Peterson, AC: Discrete Hamiltonian Systems: Difference Equations, Continued Fraction and Riccati Equations. Kluwer Academic, Dordrecht (1996)

3. Deng, $X Q$, Cheng, G: Homoclinic orbits for second order discrete Hamiltonian systems with potential changing sign. Acta Appl. Math. 103, 301-314 (2008). doi:10.1007/s10440-008-9237-z

4. Deng, XQ, Cheng, G, Shi, HP: Subharmonic solutions and homoclinic orbits of second order discrete Hamiltonian systems with potential changing sign. Comput. Math. Appl. 58, 1198-1206 (2009)

5. Ma, M, Guo, ZM: Homoclinic orbits and subharmonics for nonlinear second order difference equations. Nonlinear Anal. 67, 1737-1745 (2007)

6. Tang, XH, Lin, XY, Xiao, L: Homoclinic solutions for a class of second order discrete Hamiltonian systems. J. Differ. Equ. Appl. 16, 1257-1273 (2010)

7. Zhang, Q: Homoclinic orbits for a class of discrete periodic Hamiltonian systems. Proc. Am. Math. Soc. (in press)

8. Zhou, Z, Yu, JS, Chen, Y: Homoclinic solutions in periodic difference equations with saturable nonlinearity. Sci. China Math. 54, 83-93 (2011)

9. Chen, WX, Yang, MB, Ding, YH: Homoclinic orbits of first order discrete Hamiltonian systems with super linear terms. Sci. China Math. 54, 2583-2596 (2011)

10. Lin, $X Y$, Tang, $X H$ : Existence of infinitely many homoclinic orbits in discrete Hamiltonian systems. J. Math. Anal. Appl. 373, 59-72 (2011)

11. Ma, M, Guo, ZM: Homoclinic orbits for second order self-adjoint difference equations. J. Math. Anal. Appl. 323(1), 513-521 (2006)

12. Tang, $\mathrm{XH}$, Chen, J: Infinitely many homoclinic orbits for a class of discrete Hamiltonian systems. Adv. Differ. Equ. 2013, 242 (2013). doi:10.1186/1687-1847-2013-242

13. Tang, $\mathrm{XH}$, Lin, $\mathrm{XY}$ : Homoclinic solutions for a class of second order discrete Hamiltonian systems. Acta Math. Sin. 28 609-622 (2012)

14. Tang, $\mathrm{XH}$, Lin, $\mathrm{XY}$ : Infinitely many homoclinic orbits for discrete Hamiltonian systems with subquadratic potential. J. Differ. Equ. Appl. 19, 796-813 (2013)

10.1186/1687-1847-2014-73

Cite this article as: Wang: New potential condition on homoclinic orbits for a class of discrete Hamiltonian systems. Advances in Difference Equations 2014, 2014:73

\section{Submit your manuscript to a SpringerOpen ${ }^{\ominus}$ journal and benefit from:}

- Convenient online submission

- Rigorous peer review

Immediate publication on acceptance

- Open access: articles freely available online

- High visibility within the field

- Retaining the copyright to your article 